\title{
An investigation of ultrasound methods for the assessment of sex and age from intact human teeth
}

\author{
Robin N. M. Feeney* \\ Department of Anthropology, The Ohio State University, Columbus, Ohio
}

\begin{abstract}
Determining sex and age in human remains is necessary to achieve positive identification of individuals in forensic settings, and to provide data required for demographic analyses in archaeological samples. Due to their denser mineralization, teeth may be better preserved than other skeletal elements, which are often fragmentary and poorly preserved. This work is the first to investigate the use of ultrasound methods to accurately, objectively, and non-destructively assess sex and estimate age of human skeletal remains from intact teeth. An ultrasound imaging system using pulse-echo technique and nominal frequency $(3.5 \mathrm{MHz})$ longitudinal waves was developed for application on teeth. Mechanical and acoustic properties of teeth were examined to explore their relationship with the interaction of ultrasound wave propagation.
\end{abstract}

Determining sex and age in human remains is necessary to achieve positive identification of individuals in forensic settings and to provide data required for demographic analyses in archaeological samples. Due to their denser mineralization, teeth are generally better preserved than other skeletal elements, which can be fragmentary or poorly preserved. As a result, biological and forensic anthropologists are continually seeking accurate, objective, and nondestructive methods for assessing sex from dental remains.

External tooth dimensions have been used to determine sex in contemporary (Garn et al., 1977; Margetts and Brown, 1978; Potter et al., 1981; GarciaGodoy et al., 1985; Kieser et al., 1985a,b; De Vito and Saunders, 1990) and archaeological (Lunt, 1969; Ditch and Rose, 1972; Scuilli et al., 1977; Owsley and Webb, 1983; Stermer Beyer-Olsen and Alexanderson, 1995; Teschler-Nicola and Prossinger, 1998) samples using population-specific discriminant function formulae. However, these functions are typically more descriptive, reflecting sex differentiation of the population, than

Editor's note: Ms. Feeney's paper was awarded "First Prize" for 2005 in the Albert A. Dahlberg student research competition sponsored by the Dental Anthropology Association.
Experiments were conducted to determine differences in wave propagation in teeth from individuals of different ages and sex, both permanent and deciduous. Consistent differences in integral acoustic response patterns in the different teeth were found. It is concluded that pulse-echo ultrasound is a viable non-destructive technique to yield integral acoustic characteristic properties of teeth, potentially useful for assessing sex and estimating age, and resolving minimum numbers of individuals from commingled and scattered remains. Information developed from this study will be significant to future research insofar as it introduces a new potential method that is nondestructive, fast, and easy to administer in situ. Dental Anthropology 2005;18:2-11.

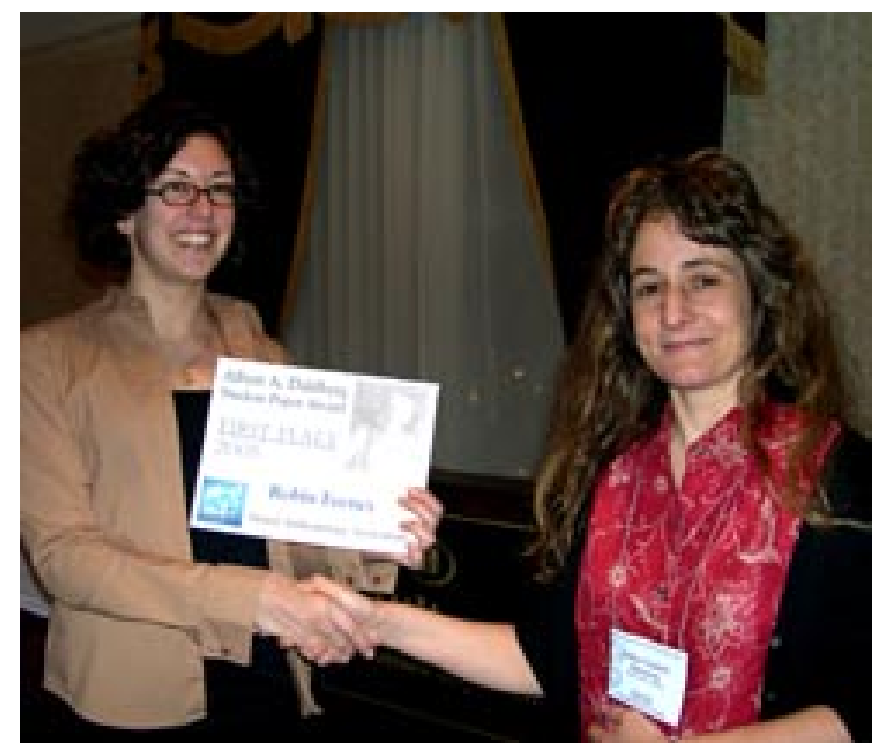

Robin Feeney (left) receiving Dahlberg Award from DAA President Debbie Guatelli-Steinberg.

*Correspondences to: Robin N. M. Feeney, Department of Anthropology, 244 Lord Hall, 124 West 17th Avenue, The Ohio State University, Columbus, Ohio 43210. E-mail:feeney.34@osu.edu 
predictive. Furthermore, interproximal attrition precludes obtaining proper measurements (TeschlerNicola and Prossinger, 1998). Moreover, the degree of human sexual dimorphism in crown size is not pronounced and actual size differences in individual teeth between sexes are small (Hillson, 1996), relative to observer error.

Thickness of enamel and dentin from sections (Moore, 1998) and radiographs (Stroud et al., 1994; Harris et al., 2001; Zilberman and Smith, 2001) of teeth also have been used to investigate sexual dimorphism. Despite the larger size of male teeth, both relatively and absolutely, enamel thickness between the sexes does not differ significantly (Stroud et al., 1994; Moore, 1998; Harris et al., 2001; Zilberman and Smith, 2001). The results of these studies all found males to possess relatively more dentin and pulp than females. However, measurements taken from radiographs require correction for magnification (Grine et al., 2001). Alternatively, sectioning teeth is destructive.

Additionally, despite the fact that the deciduous dentition demonstrates significant sexual dimorphism (Moss and Moss-Salentijn, 1977; Black, 1978; Margetts and Brown, 1978; De Vito and Saunders, 1990; Harris, 1994) and that no reliable means of determining sex from juvenile skeletal remains exists, researchers typically have limited their studies to the permanent dentition.

In estimating dental age, it is established that the calcification stages of teeth are a superior indicator of chronological age than are the eruption status of teeth or even the ossification of the skeleton (Gleiser and Hunt, 1955; Lewis and Garn, 1960). Furthermore, there is a higher correlation between dental age and chronological age than there is between dental age and skeletal age (Demirjian, 1978). In addition, dental mineralization appears to be well buffered, being comparatively unaffected by nutritional (Garn et al., 1965a) and endocrine status (Garn et al., 1965b) that impact on the tempo of an individual's bony maturation.

Various other methods have been employed to estimate age from the dentition. Several histological methods of age estimation for adult remains exist that examine cementum annulation layers (Stott et al., 1982; Wittwer-Backofen et al., 2004) and secondary dentin deposits (Gustafson, 1950), but these are destructive of the tooth sample. Furthermore, morphological methods, including dental attrition (Brothwell, 1989)

\footnotetext{
${ }^{1}$ Acoustic impedance: the opposition to the flow of sound through a surface in unit area when a wave meets the interface between two media (Wells, 1977).

2Elastic Modulus: or Young's modulus is the resistance of a material to stress: the greater the resistance, the greater the stiffness or modulus of elasticity (Wells, 1977).
}

and periodontal regression and root translucency (Lamendin et al., 1992; Prince and Ubelaker, 2002), carry with them an element of subjectivity.

After the completion of tooth development, it is increasingly difficult to assess age accurately (Xiaohu et al., 1992). Studies by Philippas (1961) and Philippas and Applebaum (1966), on a large sample of modern permanent teeth from both sexes, found that with advancing age, irregular secondary dentin progressively fills the pulp chamber of the entire crown and root inward toward the pulp, in a natural process that occurs faster in the early years and more slowly later on, regardless of occlusal wear (attrition and abrasion). Changes in the structure of teeth with age, by measuring the increase in mineral content, seem to possess potential as a nondestructive method of estimating age from teeth.

The present pilot study is a first step in investigating the principles, limitations, and possibilities of using a pulse-echo ultrasound method to non-destructively, accurately, and objectively assess sex and estimate age of human skeletal remains from intact teeth. The study of mechanical and acoustic properties of enamel and dentin has been restricted to diagnostic ultrasound in the field of endodontics. The present work examines the mechanical and acoustic properties of teeth and explores their relationship with the interaction of ultrasound wave propagation. Experiments were performed to determine differences in wave propagation in teeth from different ages and sex, in both permanent and deciduous teeth, with other experimental factors. A description of the design and implementation of the dental ultrasound system developed in this research is presented, along with information for future development and improvement of the system and methodology.

\section{PULSE-ECHO TECHNIQUE}

Ultrasound are high frequency sound waves above the range of audible frequencies; above $20 \mathrm{kHz}$ (Hussey, 1975). The analysis of sound wave disturbance along its propagation path forms the basis of ultrasound testing. Ultrasound wave motion creates disturbances in motion carrying energy, giving rise to wave properties, including the reflection a wave front, characteristic impedance ${ }^{1}$, attenuation, and absorption, whose behavior produce acoustic phenomena (Wells, 1977). As a wave propagates from one medium to another, some of the wave energy is reflected back from the interface while the rest are transmitted through the second medium. If the ultrasound wave travels through more than two media, reflections will also occur at subsequent interfaces. The phenomenon of wave propagation in dental hard tissues is a complex interplay between the parameters of the sound wave and the characteristics of the medium. Ultrastructural differences in enamel and dentin give rise to differences in mechanical properties (hardness and elastic modulus ${ }^{2}$ ) of these 


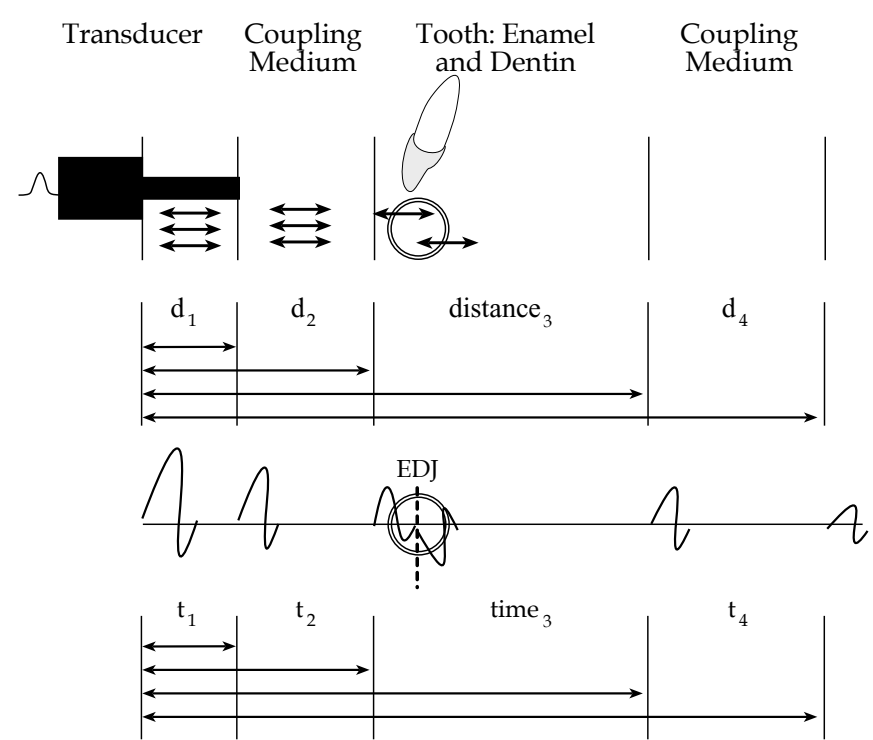

Fig. 1. Schematic illustration of the ultrasound pulse-echo technique. The circled regions indicate phase inversion. Orientation of the tooth relative to the transducer sensor beam is important because ultrasound waves will especially deviate when meeting a curved surface. Accordingly, specimens were placed in the test chamber with the most flat crown surface facing the sensor tip. Because only single-rooted teeth were studied, this commonl was the labial or buccal surface of the tooth.

tissues and result in marked difference in their sound characteristics (acoustic velocity and impedance). As a result of these differences, when ultrasound propagates from the surface of the tooth enamel into dentin, there are marked reflections at the enamel-dentin interface. Figure 1 diagrams the ultrasound pulse-echo technique, showing echoes corresponding to layers along the ultrasound beam path. Pulse 1 corresponds to the transducer surface, pulse 2 to the front surface of the coupling medium, pulse 3 to the tooth surface, and pulse 4 to the back surface of the coupling medium. Note that the second, third, and fourth echo travel the

TABLE 1. Summary of the physical properties of acoustic media used in the experiments

\begin{tabular}{lccc}
\hline Medium & $\begin{array}{c}\text { Sound } \\
\text { speed }\left(\mathrm{m} / \mathrm{s}^{-1}\right)\end{array}$ & $\begin{array}{c}\text { Density } \\
\left(\mathrm{kg} / \mathrm{m}^{3}\right)\end{array}$ & $\begin{array}{c}\text { Characteristic } \\
\text { impedence } \\
\left(\times 10^{6} \mathrm{~kg} \mathrm{~s}^{-1} \mathrm{~m}^{-2}\right)\end{array}$ \\
\hline Water & 1495 & 1000 & 1.5 \\
Honey & 2000 & 1500 & 3.0 \\
Glycerol & 1923 & 1173 & 2.3 \\
Enamel & 6000 & 2850 & 17.0 \\
Dentin & 4000 & 2150 & 8.6 \\
Steel & & & 4.6 \\
Transducer & 5920 & 7850 & 4 \\
\hline
\end{tabular}

distance twice. As shown in Figure 1, with pulse-echo ultrasound imaging different surfaces that lie one behind the other can be separated. For that reason, pulse-echo information is valuable for measuring thickness of materials, such as enamel and dentin.

Pulse-echo patterns can also be used to analyze material properties of a tooth that are related to its acoustic characteristic impedance (Wells, 1977). If an ultrasound wave passes from a medium of higher impedance (e.g., enamel) to one with lower impedance (e.g., dentin), phase inversion (where the phase of a reflected wave is reversed) occurs at the EDJ. The effect of phase inversion is illustrated in Figure 1 as an inverted echo at the EDJ, occurring after the echo that corresponds to the enamel surface.

\section{MATERIALS AND METHODS}

\section{Tooth sample}

A sample $(n=18)$ of clinically extracted permanent and deciduous incisor, canine, and premolar teeth from individuals of European and continental and subcontinental Indian descent was collected. The sample was chosen according to specific criteria: only those teeth that presented no or insignificant pathology (noninvasive carious lesions) and little wear were used. To avoid potentially complicated acoustic response signals, only single rooted teeth were used. Actual sex and age of the individuals were known.

\section{Ultrasound system}

Components of the ultrasound system developed in this research are presented in Figures 2 and 3. The data acquisition system consists of a single nominal frequency $(3.5 \mathrm{MHz})$ longitudinal wave transducer, steel testing cell, a pulser/receiver cable, and an amplifier (Panametrics $200 \mathrm{MHz}$ computer controlled pulser/ receiver, model 5900PR). The data processing system consists of an oscilloscope with a disk data storage medium (LECROY $500 \mathrm{MHz}$ oscilloscope, model 9350AM), a mainframe computer, and data processing software (MATLAB version 12.0).

The transducer (Fig. 3) is a custom-made product by Phoenix Inspection Ltd. (product number 976307). Electrical pulses applied by the amplifier and passing through the pulser/receiver cable attached to the transducer will transform into sonic energy in the form of an ultrasound wave that will propagate into the target media. The ultrasound cell (Fig. 3) was designed with sensor openings for the transducer to screw into. In this system, the transducer operates in a closed cell, rather than acting as a probe. In the center of the cell is the testing chamber, in which the tooth specimen is suspended during testing. The test chamber and transducer sensor tip were specially designed to accommodate the small size of human teeth in order to 


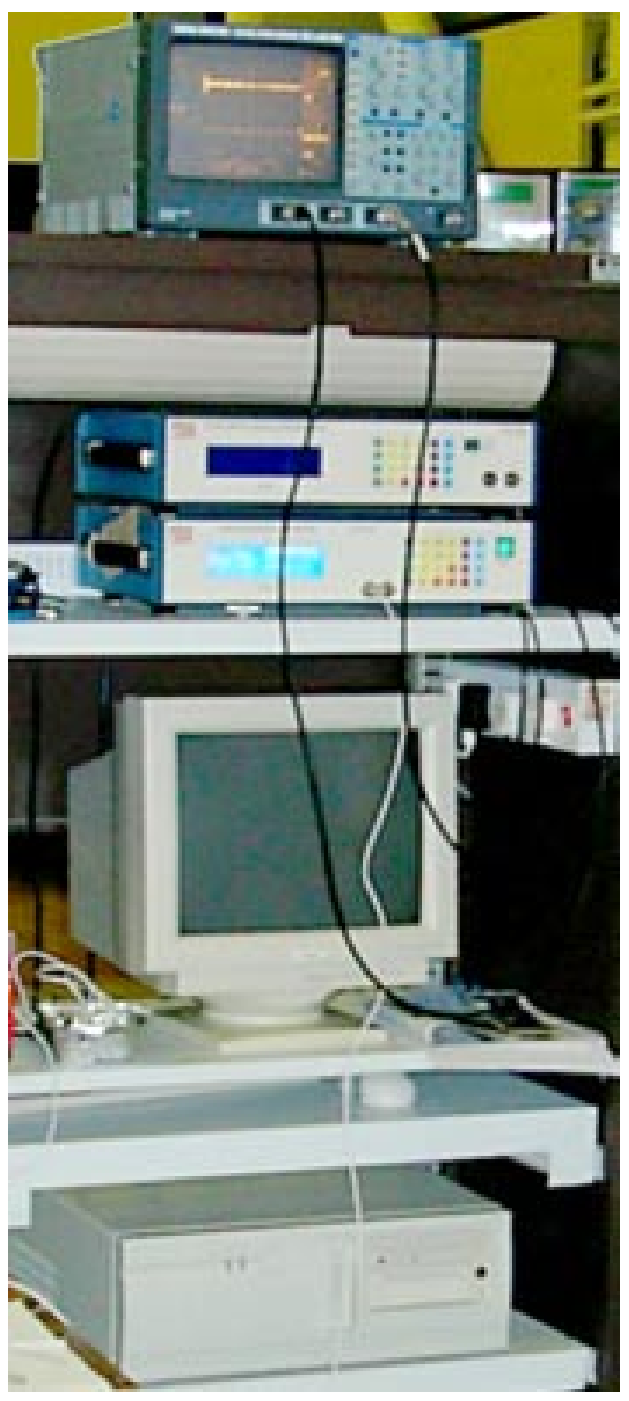

Fig. 2. Photograph of the oscilloscope, amplifier, pulser/receiver cable, and mainframe computer.

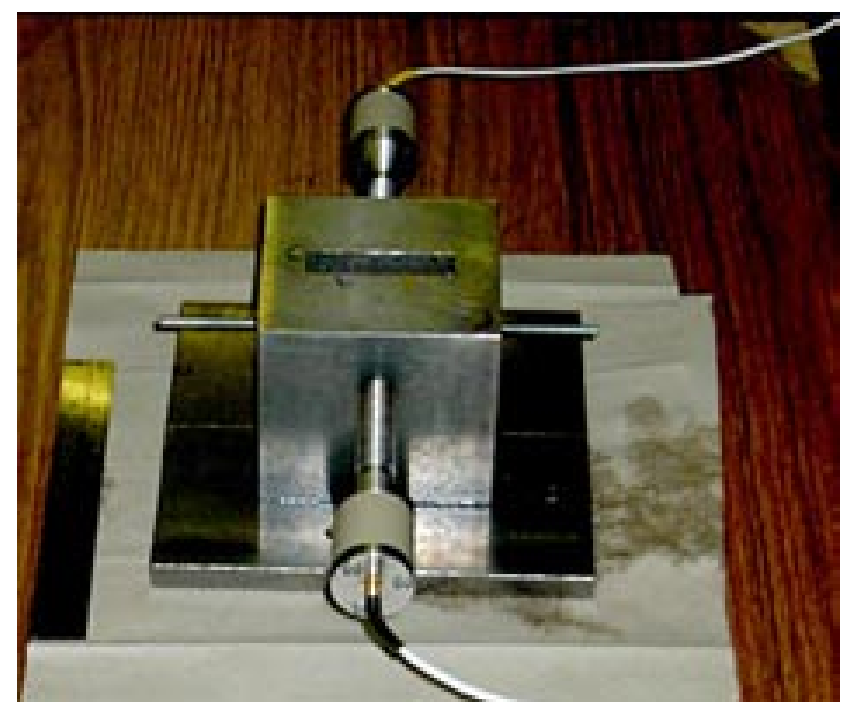

Fig. 3. Photograph of the ultrasound testing cell and transducer. provide a better quality signal.

\section{Experimental investigations}

In order to achieve a superior acoustic response in teeth, additional experiments were conducted using different coupling media and specimens with different degrees of saturation. After these preliminary investigations were completed, a series of ultrasound tests were conducted on the tooth sample to obtain information on teeth from different ages and sex, in both the permanent and deciduous dentitions. Tests were also performed on different teeth from the same individual to explore the ultrasonic response to natural variation in teeth. In order for the results for each type of test to be comparable, the tooth specimens were matched for similar traits, such as sex, age, tooth class (incisor; canine; premolar), tooth type (first, second, third; upper, lower), and permanent versus deciduous throughout the experiments. Systematic tests disclosed no significant inter- and intra-observer error and disclosed significant reproducibility of the system.

\section{RESULTS AND DISCUSSION}

\section{Dental ultrasound}

It is difficult to find a suitable material to couple enamel. In theory, mercury (Reich et al., 1967) would be a good couplant to match the high characteristic impedance of enamel because of its fluidity and high density. However, its toxicity renders it unsafe for use and its high surface tension may lead to incomplete saturation in the presence of air-filled voids in the tooth. A coupling medium of relatively high density and high velocity was required to transfer energy from the transducer to the tooth. Typically, water is used as a couplant for teeth (Lees, 1968; Ng et al., 1989; Yang, $1991 ; \mathrm{Ng}, 1993)$. However, the characteristic impedance of water is nowhere near that of enamel or dentin (Table 1 ), meaning that the coupling of ultrasound in dental tissues is very inefficient. This contrasts to situations involving soft tissues, where the characteristic impedance is similar to water and so energy losses are small. Preliminary experiments using distilled water resulted in a very weak ultrasonic response from the tooth, confirming significant acoustic energy loss.

Pure honey is a high velocity and dense fluid. In theory, because its acoustic impedance is closer to enamel than water (Table 1), it should couple better with the tooth, though it is viscous and has poor wetting properties. Preliminary experiments found pure honey to couple very well with teeth. As far as could be determined, no other investigator has used honey as a coupling medium for teeth. Glycerol, a less viscous medium than honey (Table 1), was also examined and was found to have coupling characteristics comparable to honey. Because honey is readily available and 
inexpensive, it was chosen as the coupling medium.

Saturation of the teeth was deemed necessary to eliminate air-filled voids resulting from the decay of tissues after extraction. Exclusion of air from the path of the ultrasound beam is important because air blocks the transmission of ultrasound. The effects of hydration on the acoustic properties of mineralized tissues have been previously reported (Smirnow and Wolfe, 1967; Barber et al., 1969). The effects on the acoustic properties of teeth saturated for over 24 hours are not known. In this research, teeth were saturated in distilled water for a period of one hour and for several months. Notable differences were found. Teeth saturated for one hour demonstrated improved reflections compared to teeth saturated for several months. To a certain extent, the reflection qualities of a material depend on its material properties: a tooth with good structural integrity will reflect with more scatter than one of poorer structural integrity. Therefore, with longer periods of saturation, the internal structures of the teeth are likely to become less distinguishable ultrasonically due to decay. This finding is of significance, as it will assist in the development of procedural methods for future dental ultrasound testing.

\section{Dental tissue thickness determination}

In pulse-echo ultrasound, it is possible to calculate the distance (i.e., linear measurements of tissue thickness) of enamel and dentin using the echoes corresponding to the enamel surface and the enamel-dentin interface. infact, on account of sensitivity problems and poor resolution of the image, it was not possible to detect these various interfaces. However, by working backwards: if the thickness of the enamel and dentin and the time taken for sound to travel through the honey coupling fluid, enamel, and dentin are known, time differences between echoes can be calculated (Appendix). This information is useful for approximating the enamel surface and the enamel-dentin junction (EDJ) echoes (Fig. 4). Ultrasound measurements were taken of the fluid filled chamber with no tooth (the reference measurement) and with a specimen present (Fig. 4). The specimen was removed from the test chamber and sectioned with a microtome in the same plane that the beam passed through in the tooth during ultrasound testing. Maximum thickness of enamel and dentin were measured from a photograph taken using a video microscope. Using these thickness measurements of enamel and dentin and the measured distance of the honey from the transducer sensor tip to the surface of the tooth in the test chamber, the times traveled by the ultrasound pulse in enamel, dentin, and in the honey coupling fluid were calculated (Appendix). With known time of flight of the ultrasonic pulse in these various media, the enamel surface and EDJ echoes in the time history plot (Fig. 4) could be approximated. At present, accuracy determinations are not possible
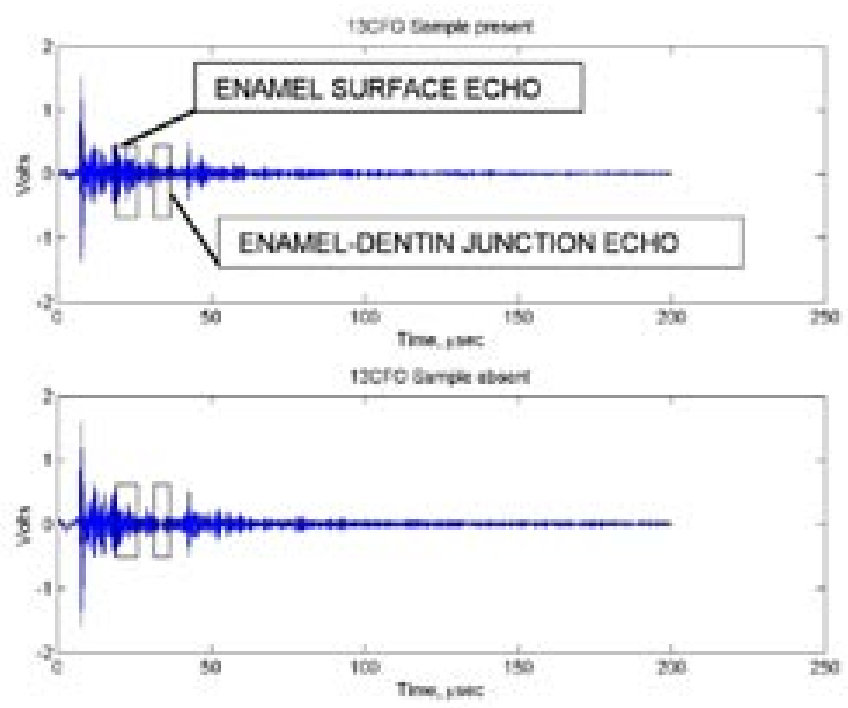

Fig. 4. Time history plot of the ultrasound response in a tooth (above) and in an empty chamber (below).

using the pulse-echo method developed in this research. Because the ultrasonic response is integral (i.e., averaged values), it comes from different areas of the tooth, thus obscuring the reflections from the different tissue layers. A higher frequency transducer with better spatial resolution would focus ultrasound energy on a limited area of the tooth and provide better image quality, allowing more accurate determination of the various interfaces, and hence determinations of enamel and dentin thicknesses.

\section{Material properties}

In addition to calculating distances in materials useful for measuring tissue thickness, pulse-echo ultrasound is useful for detecting differences in properties in materials (Wells, 1977). On account of this, structural and compositional differences in a tooth may be detected by differences in wave propagation in enamel and dentin, which are the result of different acoustic behaviors related to the ultrastructure of these dental hard tissues.

Differences in wave propagation are measured in wave frequency, amplitude (sound intensity pressure), wavelength, wave speed, and energy transmission and intensity. The determination of these various measurements was not performed in this work. The sheer volume of data and specialized knowledge required to process the results make this an inappropriate method for use by anthropologists. Instead, a method that offers fast and easily interpretable results by examining patterns in wave propagation was undertaken. Although measured differences in wave propagation offer better scientific judgment of results, waveform plots demonstrating integral acoustic intensity at a given time and frequency window are used to detect 
patterns of wave propagation between different teeth (Figs. 5-8).

The waveform plots (Figs. 5-8) are based on a time-frequency dependency of reflected strength of the ultrasound field in a tooth. The reflection qualities in the ultrasound field depend, to a certain extent, on the material properties of the hard tissues of the tooth. Peaks and troughs in reflection packets, represented by clusters of dark spots and illuminations, characterize the strength of the reflections. When acoustic energy is lost due to absorption (e.g., from air pockets in the tooth), very little of the signal has gone into the dental tissues, and this is represented by the dark spots in the images. In a time-frequency domain, improved reflections are represented by illuminated lines and patterns. The strength of the illuminations is characterized by a continuum of color, with lighter colors indicating higher intensity and darker colors indicating lower intensity. Beginning at approximately 140 microseconds, the tail ends of the plots are characterized by significant noise from reflections in the transducer waveguide and should not be interpreted.

\section{Age differences}

The progressive infilling of the root of a tooth with secondary dentin is correlated with age (Gustafson, 1950). Since the propagation of an ultrasound wave is affected by the material properties of a tooth, it is assumed that increased root dentin sclerosis in older individuals will provide different integral patterns of ultrasound wave propagation.

Teeth from individuals of varying ages were examined. Figure 5 represents the response pattern of lower central incisor teeth from a 12 and 45 year-old and premolar teeth from a 46 and 70 year-old. In general, a trend in increased reflection intensity can be observed in the teeth from younger to older individuals, revealed by

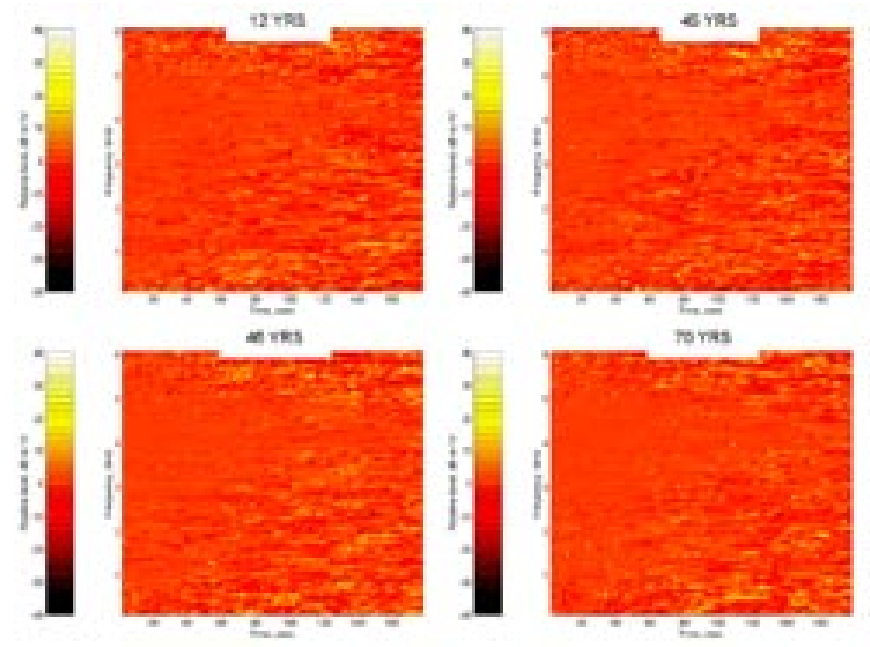

Fig. 5. Comparison of teeth from different aged individuals. increased illuminations and very few dark spots (Fig. 5). While better reflection qualities were observed in teeth from older individuals, likely due to advancing sclerosis, no obvious differences in wave propagation patterns were detected between similar teeth from individuals of different ages. New information, however, may become available with further data processing, by documenting the frequencies and times when a signal is present and performing statistical tests for pattern recognition.

\section{Sex differences}

Teeth from both sexes were examined and an apparent difference in the pattern of reflected energy intensity between the sexes was observed: a more uniform and band-like pattern was found to exist in female teeth, under 130 microseconds and in the 3-5 $\mathrm{MHz}$ frequency range, compared to males who exhibited a more distributed and irregular pattern in the whole of the frequency range (Fig. 6). Although discriminatory patterns of ultrasound wave propagation have been observed in teeth from different sexes it is difficult to determine exactly what these differences are. Most likely, these patterns are attributed to larger proportions of dentin in male teeth compared to females Stroud et al., 1994; Moore, 1998; Harris et al., 2001; Zilberman and Smith, 2001). Because dentin is less heavily mineralized than enamel, energy is more likely to attenuate in this tissue, resulting in the distributed and irregular pattern of reflected acoustic energy found in the male teeth. A larger sample is required to determine if these sexspecific patterns are statistically significant.

\section{Permanent versus deciduous teeth}

Original tests were undertaken to investigate ultrasound wave propagation in deciduous teeth. Figure 7 presents the ultrasonic response for a lower right

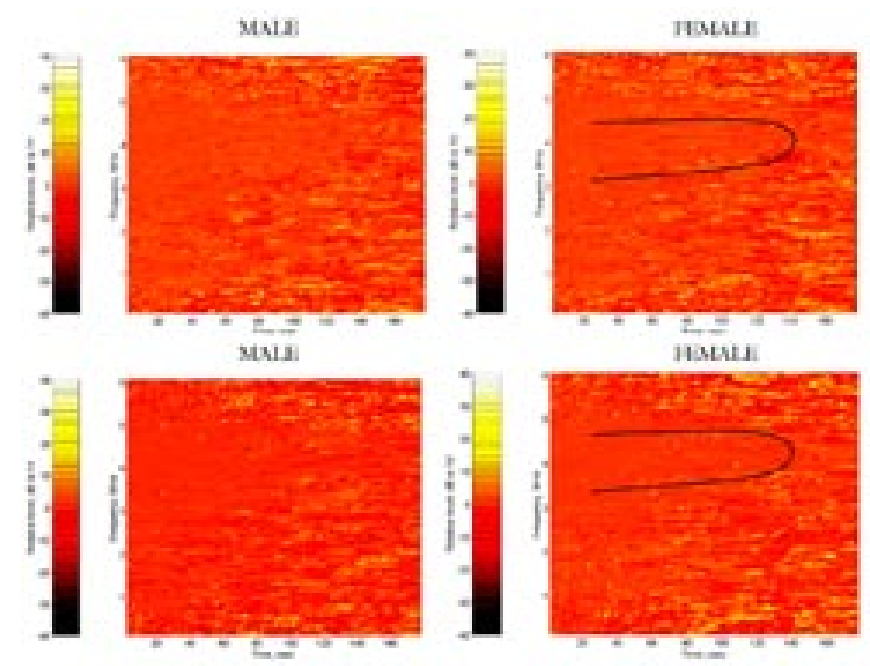

Fig. 6. Comparison of premolar teeth between different sexes. 
deciduous canine and a lower left lateral deciduous incisor, compared with two permanent teeth of the same type, respectively. The distribution of acoustic energy arriving in microseconds and at frequencies in megahertz is markedly different between permanent and deciduous teeth. The deciduous teeth demonstrate a more uniform distribution of acoustic energy than permanent teeth, particularly below 90 microseconds and in the frequency range of $2-5.5 \mathrm{MHz}$ (Fig. 7). This observed pattern in deciduous teeth, compared to their permanent counterparts, is attributed to differences in their dynamic properties. Apart from the paper by Mahoney and associates (2000) who determined that hardness and modulus of elasticity in deciduous enamel and dentin are within the range reported for permanent teeth, research on the mechanical and acoustic properties of deciduous teeth is lacking. Consequently, only speculations about the source of the wave propagation pattern observed in deciduous teeth (Fig. 7) can be made. For example, it is possible that because enamel in deciduous teeth is much thinner than in permanent teeth, the teeth are overall less hard, allowing for better coupling with the fluid medium, resulting in more energy being absorbed in the timefrequency window noted above.

The acoustic response pattern in deciduous teeth (Fig. 7) is notably similar to the patterns found in female teeth (Fig. 6), which display a uniform and clustered pattern of reflected energy in a high frequency range. It is possible that the ultrasonic response revealed in the deciduous specimens (Fig. 7) is attributed to the sex of these teeth: they are from females. Further investigation using a larger sample of deciduous teeth, however, is needed to confirm whether a unique pattern exists for deciduous teeth or whether the patterning is the result of other variables, such as sex.

\section{Natural variation in teeth}

Four deciduous canine teeth from the sameindividual were tested to investigate whether differences in natural variation in teeth could be detected ultrasonically (Fig. 8). Despite the difference in the reflected strength of the ultrasound field in the lower right specimen (due to an inconsistent saturation time) compared to the other three specimens (Fig. 8), the integral acoustic characteristic patterns were found to be remarkably similar. The most important finding in this experiment was that canine teeth from the same individual presented very similar patterns in the characteristic response of the ultrasound field. Further testing on a larger sample and improved data processing methods are required to determine if this finding is significant. If so, it may be possible, using the ultrasound method developed in this research, to discriminate between different individuals in commingled or scattered remains from their acoustic response patterns. Furthermore, it would be valuable

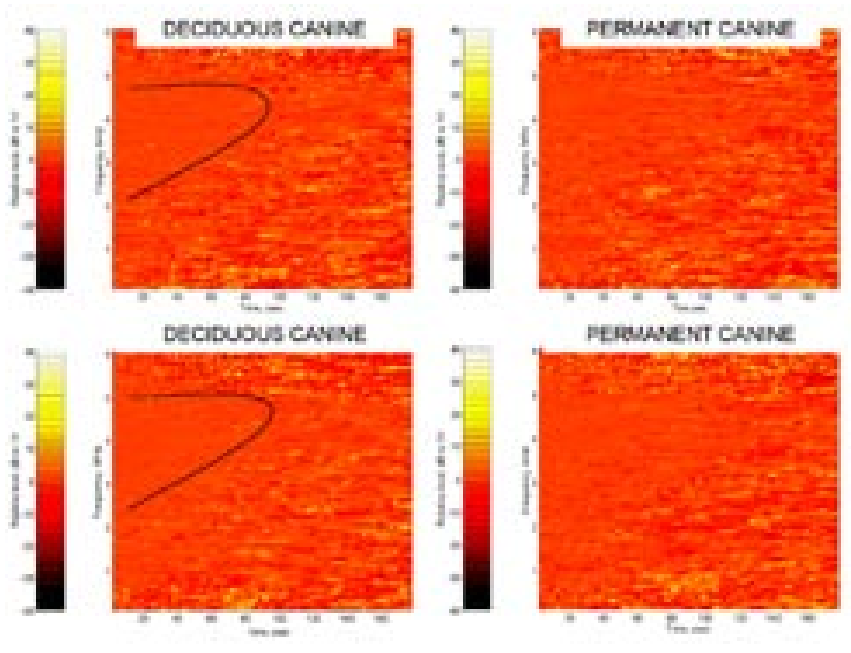

Fig. 7. Comparison between permanent and deciduous incisor and canine teeth.

to compare whether identical teeth from different individuals have different energy reflection patterns to test if the pattern in unique to individuals or to tooth class. This information may be of additional use in discriminating between individuals in mixed contexts.

\section{Limitations and future directions}

The sample $(\mathrm{n}=18)$ is notably small and the discriminatory effectiveness of the results is questionable. Although sample size was modest, the study provided insight into the possible applications and intricacies of using pulse-echo ultrasound methods for anthropological study of teeth.

The values of acoustic impedance of the steel transducer, the honey couplant, and enamel and dentin are all very different (Table 1 ). This impedance mismatch results in great loss of ultrasound energy at the interface

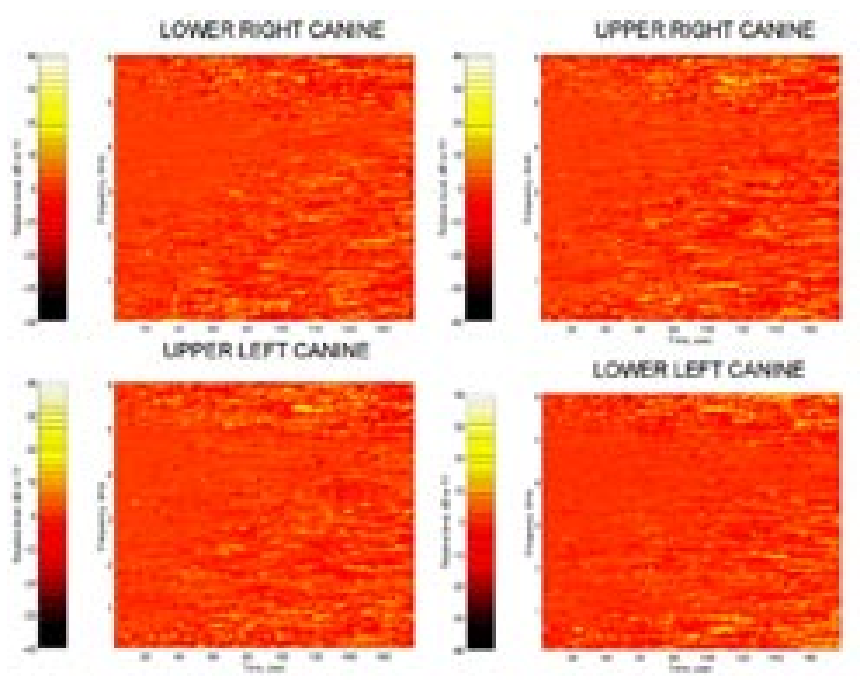

Fig. 8. Variation within canine teeth from the same individual. 
between these media. In terms of acoustic impedance, a couplant more similar to enamel than honey would be preferred. Yet a material of such high acoustic impedance would most likely have to be a solid. The nearest match to enamel is aluminum. However, there are problems associated with using a solid coupling agent. A solid metal couplant would produce noise in the form of multiple reflections that would overshadow the echoes from the interfaces under study. Furthermore, it is difficult to physically adapt a solid to the curved surface of a tooth without taking invasive measures (e.g., Lees and Barber, 1968; Barber et al., 1969), which would negate the non-destructive nature of ultrasound testing and damage the valuable tooth. One possible solution would be to use a gel contained inside a thin, soft membrane glued to the transducer, as was tried by Spranger (1971) and Fukukita et al. (1985), which appeared to adapt well to oral tissue. Apart from the considerable mismatch between the steel transducer and enamel, honey is otherwise a good couplant because it is biocompatible, readily available, and inexpensive.

The resolution of the ultrasound system is mainly dependent on the transducer characteristics. In the works by Yang (1991) and $\mathrm{Ng}$ (1993), the echo from the enamel-dentin interface was only observed on some portions of the tooth surface. Often, some areas of the tooth surface are rough or ridged and the enamel surfaces may not have been formed in parallel or in near parallel with the EDJ. These factors make the detection of the enamel-dentin interface difficult. To resolve this, a higher frequency transducer than $14.5 \mathrm{MHz}$ and 18 MHz, used by Yang (1991) and Ng (1993), respectively, should be used. Resolution problems experienced in this research may be resolved with the use of a single focused high frequency transducer. A focused high frequency transducer would offer high resolution by achieving good lateral resolution, which would enable better imaging of the curved surfaces and accommodate variation in teeth.

Of additional importance is the execution of testing and data processing. In contrast to the mounting, sectioning, and microscopic procedures normally required to obtain information about sex and age from dental remains, the operation of the ultrasound system developed in this research is both quick and straightforward. Moreover, the compact size and relatively light weight of the ultrasound testing cell and oscilloscope with data storage medium renders this system ideal for administering tests in situ, rather than in a specialized laboratory. The methods for processing the data are, however, protracted. At present, specialized knowledge of software designed to process ultrasound data and to transform it into interpretable plots is essential, as is experience interpreting the results. However, the dental ultrasound system is in the pilot stage of development. With further work, more simplified software can be developed for straightforward use and simple interpretation for use by non-specialists.

Apparent differences in integral acoustic characteristic patterned responses were observed in different types of teeth in these experiments. Further processing of data, such as documenting the frequencies and times when a signal is present and performing statistical tests for pattern recognition to find significant patterns, may offer further information on which to base interpretations. Additionally, the development of a prediction model for wave propagation in teeth (e.g., Ghorayeb et al., 1998; Ghorayeb, 2001) may further facilitate interpretations. Nevertheless, although the results do not provide a statistical basis for definitive judgment, consistent differences have been observed in the patterns of the time of arrival of the ultrasound energy in microseconds and in frequencies at megahertz in teeth, particularly in teeth from different sexes, deciduous teeth, and in identical types of teeth from the same individual.

\section{SUMMARY AND CONCLUSIONS}

This paper describes original work conducted on the use of pulse-echo ultrasound as a means of nondestructively assessing sex and estimating age in skeletal remains from intact teeth. The most important findings presented are: (1) non-destructive ultrasound imaging of intact human teeth, (2) non-destructive means of measuring intact enamel and dentin tissue thickness, (3) detection of consistent acoustic integral response patterns for different types of teeth, and (4) development of the understanding of how dental anisotropic materials and natural variation affect ultrasound wave propagation. Investigation of these endeavors in this research has provided additional information valuable for the future development of instrumentation and methodology for non-destructive ultrasound imaging of teeth. The dental ultrasound system has a number of feasible applications, even in its present state of development, but the accuracy of the ultrasound system and the smallest change that it can detect need to be determined. Furthermore, the development of software to assess for statistically significant patterns is required to eliminate subjectivity.

Ultrasound could be applied to the study of teeth if a thorough understanding of wave propagation in teeth were available. The difficulties are caused by the physical situation of the teeth, compounded by high-resolution requirements. The principal problems experienced in this research were: (1) small physical size of teeth, (2) complex structure and gradients in mechanical properties of teeth, (3) irregular shape of the teeth characterized by complex surface morphology, and (4) difficulty transmitting sound waves through the tooth. Although the dental ultrasound system 
developed in this research is not accurate enough at present to be of practical use, the results that have been obtained are encouraging. There exists a great deal of room for improvement of the system and ultrasound remains a promising method to tackle the problem of non-destructive study of teeth. From the abbreviated discussion of ultrasonics in this work it appears likely that significant possibilities, such as determining sex and age of remains from teeth ultrasonically, lie in the development of the instruments capable of providing precise and quantitative measurements.

\section{ACKNOWLEDGMENTS}

My gratitude is extended to Drs. Robert Pastor (Archaeological Sciences), Kirill Horoshenkov (Civil and Environmental Engineering), and Elaine Brown (Polymer Science Engineering) at the University of Bradford, UK, for their valuable assistance and for providing access to necessary equipment and facilities. In addition, I would like to thank the dental surgeons at the Manningham Lane Dental Center and Mark Edwards Dental Practice (West Yorkshire, UK) for providing me with the sample.

\section{LITERATURE CITED}

Barber FE, Lees S, Lobene RR. 1969. Ultrasonic pulseecho measurements in teeth. Arch Oral Biol 14: 745-760.

Black TK. 1978. Sexual dimorphism in the toothcrown diameters of the deciduous teeth. Am J Phys Anthropol 48:77-82.

Brothwell DR. 1989. The relationship of tooth wear to aging. In: Iscan MY, editor, Age markers in the human skeleton. Springfield: C. C. Thomas, p 303316.

Demirjian A. 1978. Dentition. In: Falkner F, Tanner JM, editors, Human growth. Volume 2. New York: Plenum Press, p 413-444.

De Vito C, Saunders SR. 1990. Discriminant function analysis of deciduous teeth to determine sex. J Forensic Sci 35:845-858.

Ditch LE, Rose JC. 1972. A Multivariate dental sexing technique. Am J Phys Anthropol 37:61-64.

Fukukita H, Yano T, Fukumoto A, Sawada K, Fujimasa T, Sunada I. 1985. Development and application of an ultrasonic imaging system for dental diagnosis. J Clin Ultrasound 13:597-600.

Garcia-Godoy F, Michelen A, Townsend G. 1985. Crown diameters of the deciduous teeth in Dominican mulatto children. Hum Biol 57:27-31.

Garn SM, Lewis AB, Kerewsky RS. 1965a. Genetic, nutritional, and maturational correlates of dental development. J Dent Res [supplement] 44:228-243.

Garn SM, Lewis AB, Blizzard RM. 1965b. Endocrine factors in dental development. J Dent Res [supplement] 44:243-258.

Garn SM, Cole PE, Wainwright RL, Guire KE. 1977. Sex discriminatory effectiveness using combinations of permanent teeth. J Dent Res 56:697.

Gleiser I, Hunt EE. 1955. The permanent mandibular first molar: its calcification, eruption and decay. Am J Phys Anthropol 13:253-284.

Ghorayeb SR. 2001. Modeling of ultrasonic wave propagation in teeth using PSpice: a comparison with finite element models. IEEE T Ultrason Ferr 48: 1124-1131.

Ghorayeb SR, Xue T, Lord W. 1998. A finite element study of ultrasonic wave propagation in a tooth phantom. J Dent Res 77:39-49.

Grine FE, Stevens NJ, Jungers WL. 2001. An evaluation of dental radiograph accuracy in the measurement of enamel thickness. Arch Oral Biol 46:1117-1125.

Gustafson G. 1950. Age determination on teeth. J Am Dent Assoc 41:45-54.

Harris EF. 1994. Factor analytic analysis of the deciduous dentition of American Blacks [abstract]. Am J Phys Anthropol [supplement] 18:102.

Harris EF, Hicks JD, Barcroft BD. 2001. Tissue contributions to sex and race: differences in tooth crown size of deciduous molars. Am J Phys Anthropol 115:223-237.

Hillson S. 1996. Dental anthropology. Cambridge: Cambridge University Press.

Hussey M. 1975. Diagnostic ultrasound: an introduction to the interactions between ultrasound and biological tissues. Glasgow: Blackie and Son.

Kieser JA, Groeneveld HT, Preston CB. 1985a. An odontometric analysis of the Lengua Indians dentition. Hum Biol 57:611-620.

Kieser JA, Groeneveld HT, Preston CB. 1985b. A metric analysis of the South African Caucasoid dentition. J Dent Assoc S Afr 40:121-125.

Lamendin H, Baccino E, Humbert JF, Tavernier JC, Nossintchouk RM, Zerilli A. 1992. A simple technique for age estimation in adult corpses: the two criteria dental method. J Forensic Sci 37:13731379.

Lees S. 1968. Specific acoustic impedance of enamel and dentin. Arch Oral Biol 13:1491-1500.

Lees S, Barber FE. 1968. Looking into teeth with ultrasound. Science 161:477-478.

Lewis AB, Garn SM. 1960. The relationship between tooth formation and other maturation factors. Angle Orthod 30:70-77.

Lunt D. 1969. An odontometric study of medieval Danes. Acta Odontol Scand [supplement 55] 27:1-173.

Mahoney E, Holt A, Swain M, Kilpatrick N. 2000. The hardness and modulus of elasticity of primary molar teeth: an ultra-micro-indentation study. J Dent 28 : 589-594.

Margetts B, Brown T. 1978. Crown diameters of the 
deciduous teeth in Australian aboriginals. Am J Phys Anthropol 48:493-502.

Moore EE. 1998. Sexual dimorphism in enamel thickness in the human mandibular canine. Unpublished Master's thesis. Tennessee: University of Tennessee, Knoxville.

Moss ML, Moss-Salentijn L. 1977. Analysis of developmental processes possibly related to human dental sexual dimorphism in permanent and deciduous canines. Am J Phys Anthropol 46:407413.

$\mathrm{Ng}$ SY. 1993. Ultrasonic imaging of in vitro enamel caries. Unpublished Ph.D. Dissertation. Manchester: University of Manchester Institute of Science and Technology.

Ng SY, Payne PA, Cartedge NA, Ferguson MW. 1989. Determination of ultrasonic velocity in human enamel and dentin. Arch Oral Biol 34:341-345.

Owsley DW, Webb RS. 1983. Misclassification probability of dental discrimination functions for sex determination. J Forensic Sci 28:181-185.

Philippas GC. 1961. Influence of occlusal wear and age on formation of dentin and size of pulp chamber. J Dent Res 40:1186-1198.

Philippas GC, Applebaum E. 1966. Age factor in secondary dentin formation. J Dent Res 45:778-789.

Potter RHY, Alcazaren AB, Herbosa FM, Tomaneng J. 1981. Dimensional characteristics of the Filipino dentition. Am J Phys Anthropol 55:33-42.

Prince DA, Ubelaker DH. 2002. Application of Lamendin's adult dental aging technique to a diverse skeletal sample. J Forensic Sci 47:107-116.

Reich FR, Brenden BB, Porter NS. 1967. Ultrasonic imaging of teeth. Report of Battelle Memorial Institute, Pacific Northwest Laboratory, Richland, Washington.

Scuilli PW, Williams JA, Gugelchuk GM. 1977. Canine size: an aid in sexing prehistoric Amerindians. J Dent Res 56:1424.

Smirnow R, Wolfe M. 1967. Illumination of oral structures by pulsed ultrasound. Digest 7 th International Conference of Medical and Biological Engineering. Royal Academy Engineering Society, Stockholm, Sweden, p 326.

Spranger H. 1971. Ultrasonic diagnosis of marginal periodontal diseases. Int Dent J 21:442-455.

Stermer Bayer-Olsen E, Alexanderson V. 1995. Sex assessment of medieval Norwegian skeletons based on permanent tooth crown size. Int J Osteoarchaeol 5:274-281.

Stott GC, Sis RF, Levey BM. 1982. Cemental annulation as an age criterion in forensic dentistry. J Dent Res 61:814-817.
Stroud JL, Buschang PH, Goaz PW. 1994. Sexual dimorphism in mesiodistal dentin and enamel thickness. Dentomaxillofac Rad 23:169-171.

Teschler-Nicola M, Prossinger H.1998. Sex determination using tooth dimensions. In: Alt KW, Rosing FW, Teschler-Nicola M, editors, Dental anthropology: fundamentals, limits, and prospects. New York: Springer-Verlangwien, $\mathrm{p} 480-500$.

Wells PNT. 1977. Biomedical ultrasonics. London: Academic Press.

Wittwer-Backofen U, Gampe J, Vaupel JW. 2004. Tooth cementum annulation for age estimation: results from a large known-age validation study. Am J Phys Anthropol 123:119-129.

Xiaohu X, Philipsen HP, Jablonski NG, Pang KM, Jiazhen Z. 1992. Age estimation from the structure of adult human teeth: review of the literature. Forensic Sci Int 54:23-28.

Yang ZZ. 1991. Ultrasound surface imaging and the measurement of tooth enamel thickness. Unpublished PhD Dissertation. Manchester: University of Manchester Institute of Science and Technology.

Zilberman U, Smith P. 2001. Sex and age-related differences in primary and secondary dentin formation. Adv Dent Res 15:42-45.

\section{APPENDIX}

Calculating distances from ultrasound measurements:

1) Scale: $1 \mathrm{~mm}=18 \mathrm{~mm}$ on video microscope photograph

2) Sound Speed (c) in $\mathrm{m} / \mathrm{s}^{-1}$ :

Enamel: 6000

Dentin: 4000

Honey coupling fluid: 2000

3) Maximum thickness measurements:

Enamel: $17 \mathrm{~mm}$ on video microscope photograph $=17 / 18 \times 1 \mathrm{~mm}=0.94 \mathrm{~mm}$

Dentin: $34 \mathrm{~mm}$ on video microscope photograph $=34 / 18 \times 1 \mathrm{~mm}=1.89 \mathrm{~mm}$

Honey: $4 \mathrm{~mm}$ from transducer sensor tip to front surface of tooth in chamber

4) Time travelled $(t): t=\mathrm{Dc}$, where $\mathrm{D}=$ distance; $\mathrm{c}=$ sound speed:

Enamel: $0.94 \times 10^{-3} \times 6000=5.64 \mu \mathrm{sec}(\times 2$ for return pulse $=16 \mu \mathrm{sec}$ )

Dentin: $1.89 \times 10^{-3} \times 4000=7.56 \mu$ sec $(\times 2$ for return pulse $=15.12 \mu \mathrm{sec}$ )

Honey coupling fluid: $4 \times 10^{-3}$ x $2000=8 \mu$ sec $(\times 2$ for return pulse $=16 \mu \mathrm{sec}$ ) 